\title{
Uniform magnetic excitations in nanoparticles
}

\author{
Mørup, Steen; Hansen, Britt Rosendahl
}

Published in:

Physical Review B Condensed Matter

Link to article, DOI:

10.1103/PhysRevB.72.024418

Publication date:

2005

Document Version

Publisher's PDF, also known as Version of record

Link back to DTU Orbit

Citation (APA):

Mørup, S., \& Hansen, B. R. (2005). Uniform magnetic excitations in nanoparticles. Physical Review $B$ Condensed Matter, 72(2), 024418. https://doi.org/10.1103/PhysRevB.72.024418

\section{General rights}

Copyright and moral rights for the publications made accessible in the public portal are retained by the authors and/or other copyright owners and it is a condition of accessing publications that users recognise and abide by the legal requirements associated with these rights.

- Users may download and print one copy of any publication from the public portal for the purpose of private study or research.

- You may not further distribute the material or use it for any profit-making activity or commercial gain

- You may freely distribute the URL identifying the publication in the public portal

If you believe that this document breaches copyright please contact us providing details, and we will remove access to the work immediately and investigate your claim 


\title{
Uniform magnetic excitations in nanoparticles
}

\author{
Steen Mørup* and Britt Rosendahl Hansen ${ }^{\dagger}$ \\ Department of Physics, Building 307, Technical University of Denmark, DK-2800 Kongens Lyngby, Denmark
}

(Received 13 July 2004; revised manuscript received 16 February 2005; published 12 July 2005)

\begin{abstract}
We have used a spin-wave model to calculate the temperature dependence of the (sublattice) magnetization of magnetic nanoparticles. The uniform precession mode, corresponding to a spin wave with wave vector $q$ $=0$, is predominant in nanoparticles and gives rise to an approximately linear temperature dependence of the (sublattice) magnetization well below the superparamagnetic blocking temperature for both ferro-, ferri-, and antiferromagnetic particles. This is in accordance with the results of a classical model for collective magnetic excitations in nanoparticles. In nanoparticles of antiferromagnetic materials, quantum effects give rise to a small deviation from the linear temperature dependence of the (sublattice) magnetization at very low temperatures. The complex nature of the excited precession states of nanoparticles of antiferromagnetic materials, with deviations from antiparallel orientation of the sublattice magnetization vectors, results in a contribution to the susceptibility, which increases with increasing temperature.
\end{abstract}

DOI: 10.1103/PhysRevB.72.024418 PACS number(s): 75.10.Jm, 75.30.Ds, 75.50.Ee, 75.50.Tt

\section{INTRODUCTION}

Magnetic fluctuations in nanoparticles have been the subject of numerous studies; see, for example, Refs. 1-6 and references therein. The most dramatic effect is superparamagnetic relaxation, by which the (sublattice) magnetization direction is reversed due to thermal agitation. This can result in, for example, a disappearance of the coercivity in magnetization measurements and a collapse of the magnetic hyperfine splitting in Mössbauer spectra. It is often assumed that magnetic nanoparticles have uniaxial symmetry with magnetic energy given by

$$
E(\theta)=K V \sin ^{2} \theta
$$

The temperature dependence of the superparamagnetic relaxation time is then given by the Néel-Brown expression ${ }^{7,8}$

$$
\tau=\tau_{0} \exp \left(\frac{K V}{k_{B} T}\right) .
$$

Here, $K$ is the magnetic anisotropy constant, $V$ is the volume, $\theta$ is the angle between the (sublattice) magnetization direction and an easy direction of magnetization, $k_{B}$ is Boltzmann's constant, and $T$ is the temperature. $\tau_{0}$ is typically of the order of $10^{-9}-10^{-12} \mathrm{~s}$.

The blocking temperature, $T_{B}$, of a magnetic nanoparticle is defined as the temperature at which the superparamagnetic relaxation time is comparable to the time scale used to study the magnetic properties. At temperatures well below $T_{B}$ the magnetic properties may still be affected by magnetic fluctuations, which can influence experimental data. Although the magnetization vector cannot surmount the energy barrier separating the energy minima at $\theta=0^{\circ}$ and $\theta=180^{\circ}$, it may still fluctuate in directions close to those corresponding to the energy minima. These fluctuations, the so-called collective magnetic excitations, can be described as a uniform precession of the magnetization vector around an easy direction of magnetization in combination with transitions between these precession states. ${ }^{9,10}$ In Mössbauer spectroscopy they result in a reduction of the magnetic hyperfine splitting. Recently, it has been shown that transitions between the precession states can be studied by neutron scattering. ${ }^{11,12}$

The first calculations of the influence of collective magnetic excitations on the magnetic properties of nanoparticles ${ }^{9,10}$ were carried out using a classical model in which the magnetization direction of a particle fluctuates in a potential given by Eq. (1). For a ferromagnetic nanoparticle with a very large total spin, this is a good approximation. However, for a nanoparticle of an antiferromagnetic material the magnetic excitations are much more complex, and it is therefore not obvious that the results of the classical model can be applied.

In this paper we use an alternative approach. We consider the uniform precession states as spin waves with wave vector $q=0$ and use a spin-wave model to derive expressions for the decrease of the (sublattice) magnetization with increasing temperature. In previous theoretical papers on spin waves in nanoparticles, ${ }^{13-20}$ the magnetic anisotropy was not included in the calculations, and the energy of the uniform mode was therefore zero. This leads to unphysical results, and the uniform mode was omitted in the calculations. In our derivations we have included the anisotropy and we describe spinwave excitations with $q=0$, which have not been discussed in previous papers, even though, as we point out, the uniform excitations are the predominant magnetic excitations in nanoparticles. In the calculations we neglect surface effects, which may lead to a lowering of the magnetization, especially at temperatures which are not low compared to the Curie or Néel temperature. ${ }^{13}$ It is shown that the results of the classical model are identical to the first-order approximation of the spin-wave model for nanoparticles of both ferro-, ferri-, and antiferromagnetic materials. As in bulk material the (sublattice) magnetization, $M(T)$, of a nanoparticle decreases with temperature according to the expression

$$
M(T)=M_{0}\left(1-B T^{\alpha}\right),
$$

where $B$ is a constant. In bulk materials $\alpha=\frac{3}{2}$ for ferromagnets and $\alpha=2$ for antiferromagnets. However, for nanopar- 
ticles of both ferro-, ferri-, and antiferromagnetic materials we find that $\alpha \simeq 1$.

It is of particular interest that the two sublattices in nanoparticles of antiferromagnetic materials are not strictly antiparallel when the uniform mode is excited. We derive expressions for the average value of the absolute magnitude of the resulting magnetic moment and the related initial susceptibility.

\section{THE CLASSICAL MODEL}

In the classical model for collective magnetic excitations $^{9,10}$ it was assumed that the magnetic moment of a nanoparticle can be considered as a classical macrospin, i.e., quantization was ignored. This may be a good approximation for a ferromagnetic or ferrimagnetic particle with large total spin. For a particle with magnetic energy given by (1), the average magnetization at temperatures well below $T_{B}$ can then be calculated using Boltzmann statistics ${ }^{9}$

$$
\langle M(T)\rangle=M_{0} \frac{\int_{0}^{2 \pi} d \phi \int_{0}^{\pi / 2} d \theta \cos \theta \sin \theta \exp \left(-\frac{E(\theta)}{k_{B} T}\right)}{\int_{0}^{2 \pi} d \phi \int_{0}^{\pi / 2} d \theta \sin \theta \exp \left(-\frac{E(\theta)}{k_{B} T}\right)},
$$

where $M_{0}$ is the saturation magnetization and $\phi$ is the azimuthal angle. Here, it is assumed that superparamagnetic relaxation can be ignored, i.e., the magnetization vector remains in directions close to $\theta=0$ (or $\theta=\pi$ ) and therefore the integration over $\theta$ is performed only up to $\theta=\pi / 2$. A Taylor expansion valid in the low-temperature limit gives ${ }^{9}$

$$
\langle M(T)\rangle \simeq M_{0}\left(1-\frac{k_{B} T}{2 K V}\right) .
$$

Thus, the reduction in magnetization caused by collective magnetic excitations is given by

$$
\frac{\Delta M}{M_{0}}=\frac{k_{B} T}{2 K V} .
$$

A generalized model, which is valid for arbitrary symmetries of the magnetic anisotropy ${ }^{10}$ and in the presence of applied magnetic fields, ${ }^{21}$ has been published. The calculations showed that, irrespective of symmetry and applied magnetic fields, the first-order approximation gives a reduction in magnetization due to collective magnetic excitations, which increases linearly with temperature. If the fluctuations are fast compared to the time scale of Mössbauer spectroscopy, the magnetic hyperfine splitting of Mössbauer spectra of magnetic nanoparticles will be proportional to $\langle M(T)\rangle$. The model has been used to explain numerous Mössbauer data for magnetic nanoparticles.

Both in bulk materials and nanoparticles, the magnetization and the magnetic hyperfine field, $B_{h f}$, decrease with increasing temperature because of thermal excitation of spin waves with $q>0$. Far below the Curie or Néel temperature this decrease is very small. For example, in bulk $\alpha$-Fe,
$\alpha-\mathrm{Fe}_{2} \mathrm{O}_{3}$, and $\gamma-\mathrm{Fe}_{2} \mathrm{O}_{3}$ the decrease in (sublattice) magnetization and magnetic hyperfine field up to about $100 \mathrm{~K}$ is well below $1 \%$. For comparison, the decrease in $\langle M(T)\rangle$ due to collective magnetic excitations for a $5 \mathrm{~nm}$ particle with $K$ $\simeq 10^{5} \mathrm{Jm}^{-3}$ is of the order of $10 \%$ at $100 \mathrm{~K}$. Thus, the reduction in (sublattice) magnetization and $B_{h f}$ due to collective magnetic excitations will be predominant at low temperatures in magnetic particles with dimensions of the order of a few nanometers.

\section{SPIN-WAVE MODEL FOR FERROMAGNETIC NANOPARTICLES}

For a cubic ferromagnet with lattice constant $a$, the dispersion relation for spin waves with $a q \ll 1$ is given by ${ }^{22,23}$

$$
\hbar \omega_{q}=2 J S a^{2} q^{2}+g \mu_{B} B_{a},
$$

where $\omega_{q}$ is the angular frequency of a spin wave with wave vector $\boldsymbol{q}, J$ is the exchange coupling constant, $S$ is the spin of the atom, $g$ is the Landé factor, $\mu_{B}$ is the Bohr magneton, and $B_{a}=2 K / M_{0}$ is the anisotropy field. In nanoparticles only a finite number of $q$ values are allowed. For example, in a cubic particle of side $d$, it is a fair approximation to assume that $q_{i}(i=x, y, z)$ can take the values $q_{i}=0, \pi / d, 2 \pi$ $/ d, 3 \pi / d, \ldots,\left[\left(N_{a t}-1\right) \pi\right] / d$, where $N_{a t}$ is the number of atoms along the direction $i$. A more rigorous discussion of spin waves in nanoparticles, including the influence of surface effects on the magnetic excitations, has been published by Hendriksen et al. $^{13}$

For each spin-wave state, characterized by the wave vector $\boldsymbol{q}$, the associated spin-wave energy is given by $E_{q}$ $=\left(\left\langle n_{q}\right\rangle+\frac{1}{2}\right) \hbar \omega_{q}$, where $n_{q}$ is an integer. An increase of $n_{q}$ by 1 corresponds to a decrease of the magnetization by $g \mu_{B}$. With decreasing values of $d$, the energy difference between the mode with $q=0$ and the modes with $q \neq 0$ will increase. Therefore, in nanoparticles the modes with $q \neq 0$ will play a smaller role at low temperatures than in bulk materials. Thus, the spin wave with $q=0$ is predominant in nanoparticles as was discussed in Sec. II. Therefore, in the following we neglect the modes with $q \neq 0$.

For a nanoparticle with its magnetization direction in the energy well close to $\theta=0, n_{0}$ can assume the values $0,1,2, \ldots, N-1$. For a particle with magnetic moment $\mu$ $=M_{0} V, N=\mu / g \mu_{B}$. The average value of $n_{0}$ is given by

$$
\left\langle n_{0}\right\rangle=\frac{\sum_{n=0}^{N-1} n \exp \left(-n \frac{\hbar \omega_{0}}{k_{B} T}\right)}{\sum_{n=0}^{N-1} \exp \left(-n \frac{\hbar \omega_{0}}{k_{B} T}\right)}
$$

Introducing

$$
x=\exp \left(-\frac{\hbar \omega_{0}}{k_{B} T}\right)
$$

one finds 


$$
\left\langle n_{0}\right\rangle=\frac{d \ln \left(\sum_{n=0}^{N-1} x^{n}\right)}{d x},
$$

which leads to

$$
\left\langle n_{0}\right\rangle=\frac{1}{\exp \left(\frac{\hbar \omega_{0}}{k_{B} T}\right)-1}-\frac{N}{\exp \left(N \frac{\hbar \omega_{0}}{k_{B} T}\right)-1} .
$$

The reduction in magnetic moment of a particle due to the spin wave state $q=0$ equals

$$
\Delta \mu=\left(\left\langle n_{0}\right\rangle+\frac{1}{2}\right) g \mu_{B} .
$$

In a nanoparticle well below $T_{B}$, the last term in Eq. (11) is small and we therefore neglect it. The anisotropy field, $B_{a}$, is typically of the order of $0.1 \mathrm{~T}$ or smaller. Therefore, even around liquid helium temperature $\hbar \omega_{0}=g \mu_{B} B_{a}$ will be much smaller than the thermal energy, and one can replace the exponential in the first term of Eq. (11) with the first terms of a Taylor expansion. Equation (12) can then be written as

$$
\Delta \mu \simeq \frac{k_{B} T}{B_{a}} .
$$

This implies that the reduction in average magnetization due to excitations of the uniform mode to a good approximation is given by the classical result, Eq. (6). The calculations in this section also apply to nanoparticles of ferrimagnetic materials.

\section{SPIN-WAVE MODEL FOR NANOPARTICLES OF ANTIFERROMAGNETIC MATERIALS}

The magnetic excitations in an antiferromagnetic material are more complex than those of a ferromagnetic material. This is related to the fact that the sublattice magnetization directions are not strictly antiparallel in the excited states. $^{24,25}$

The dispersion relation for the spin waves in an antiferromagnet is given by ${ }^{23}$

$$
\hbar \omega_{q}=g \mu_{B}\left[\left(B_{E}+B_{A}\right)^{2}-B_{E}^{2}\left(1-\frac{2 q^{2} a^{2}}{z}\right)\right]^{1 / 2},
$$

where $B_{E}$ is the exchange field, $B_{A}=K / M_{S}$ is the anisotropy field, $M_{S}$ is the sublattice magnetization, and $z$ is the number of nearest-neighbor magnetic atoms.

Again, we will specifically investigate the mode with $q$ $=0$, because it is predominant in nanoparticles. Usually $B_{A}$ $\ll B_{E}$, and one then finds for the uniform mode ${ }^{24-26}$

$$
\hbar \omega_{0} \simeq g \mu_{B} \sqrt{2 B_{E} B_{A}} .
$$

This has been confirmed by numerous high-frequency ac susceptibility measurements ${ }^{22}$ and neutron scattering experiments. ${ }^{11,12}$

In several studies it has been found that nanoparticles of antiferromagnetic materials can show anomalous features. Their magnetic moments may be quite large and may show a

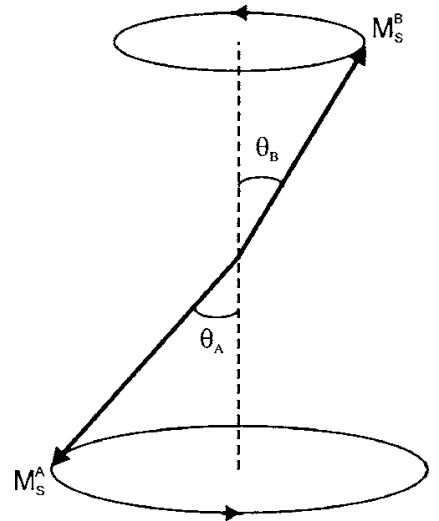

FIG. 1. Schematic illustration of the uniform mode in nanoparticles of antiferromagnetic materials. The two sublattice magnetization vectors, $\boldsymbol{M}_{S}^{\boldsymbol{A}}$ and $\boldsymbol{M}_{S}^{\boldsymbol{B}}$, precess with different precession angles, $\theta_{A}$ and $\theta_{B}$, around the easy axis of magnetization. For clarity, the difference between $\theta_{A}$ and $\theta_{B}$ is exaggerated.

temperature dependence which is not in accordance with the expected behavior. ${ }^{27-32}$ Néel $^{33}$ suggested that the large magnetic moments could be explained by uncompensated magnetic moments, but the anomalous temperature dependence has been a puzzle.

In the following, for simplicity we consider particles of antiferromagnetic particles without an uncompensated magnetic moment. We also neglect the possibility of canted surface spins. For such particles we calculate how much the sublattice magnetization is reduced at a given temperature because of the uniform mode in nanoparticles of antiferromagnetic materials. In an excited state, the two sublattice magnetization directions will form different angles $\theta_{A}$ and $\theta_{B}$ with the easy direction of magnetization, ${ }^{24-26}$ as illustrated in Fig. 1. With $B_{A} \ll B_{E}$, we have the following relation between the two angles: ${ }^{24-26}$

$$
\frac{\sin \theta_{A}}{\sin \theta_{B}} \simeq 1+\delta
$$

where

$$
\delta= \pm \sqrt{2 \frac{B_{A}}{B_{E}}} .
$$

Averaging over the fast precession around the easy direction of magnetization $\left(\omega_{0} \sim 10^{12} \mathrm{~s}^{-1}\right),{ }^{22}$ one finds that the particle will have a nonzero magnetic moment with an absolute value given by

$$
\left|\mu_{A F}\right|=M_{S} V\left|\cos \theta_{A}-\cos \theta_{B}\right| .
$$

At a transition between two neighboring precession states, the total magnetic moment of the particle is changed by the amount

$$
\Delta \mu_{A F}=M_{S} V\left[\Delta\left(\cos \theta_{A}\right)-\Delta\left(\cos \theta_{B}\right)\right],
$$

where the terms in brackets represent the difference in cosine of the precession angles of neighboring precession states.

From Eq. (16) one finds that $d \theta_{A} \simeq(1+\delta)$ $\times\left(\cos \theta_{B} / \cos \theta_{A}\right) d \theta_{B}$, and thereby 
$\Delta \mu_{A F} \simeq M_{S} V\left(-\sin \theta_{A} \Delta \theta_{A}+\sin \theta_{B} \Delta \theta_{B}\right) \simeq 2 M_{S} V \delta \Delta\left(\cos \theta_{A}\right)$.

The smallest allowed change in the total magnetic moment is $\Delta \mu_{A F}=g \mu_{B}$. We find from Eq. (20)

$$
\Delta\left(\cos \theta_{A}\right)_{\min } \equiv \xi \simeq \frac{g \mu_{B}}{2 M_{S} V \delta} .
$$

Thus, the number of precession states with $q=0$ in the energy well around $\theta=0$ is

$$
N \simeq \frac{2 M_{S} V \delta}{g \mu_{B}},
$$

i.e., the number of precession states is reduced by the factor $\delta$ compared to the case of ferromagnetic nanoparticles.

The reduction in sublattice magnetization due to the uniform mode is given by

$$
\Delta M_{S}=2\left(\left\langle n_{0}\right\rangle+\frac{1}{2}\right) \xi M_{S},
$$

where a factor of 2 has been included to take into account the degeneracy of states with positive and negative values of $\delta$. Using a relation analog to Eq. (10), we then find

$$
\Delta M_{S}=\frac{g \mu_{B}}{V \delta}\left[\frac{1}{\exp \left(\frac{\hbar \omega_{0}}{k_{B} T}\right)-1}-\frac{2 M_{S} V \delta}{g \mu_{B}} \frac{1}{\exp \left(\frac{4 K V}{k_{B} T}\right)-1}+\frac{1}{2}\right] .
$$

The relative reduction in the sublattice magnetization is given by

$$
\frac{\Delta M_{S}}{M_{S}}=\frac{\hbar \omega_{0}}{2 K V}\left[\frac{1}{\exp \left(\frac{\hbar \omega_{0}}{k_{B} T}\right)-1}+\frac{1}{2}\right]-\frac{2}{\exp \left(\frac{4 K V}{k_{B} T}\right)-1} .
$$

As in the case of ferromagnetic nanoparticles, the last term is negligible well below $T_{B}$.

Neglecting quantization effects (see Sec. V), we find for $\hbar \omega_{0} \ll k_{B} T$

$$
\frac{\Delta M_{S}}{M_{S}} \simeq \frac{k_{B} T}{2 K V},
$$

which is equivalent to Eq. (6).

\section{THERMOINDUCED MAGNETIZATION}

The large magnetization of nanoparticles of antiferromagnetic materials, which has been found in many studies, may, at least partly, be explained by uncompensated magnetic moments, but this does not explain the anomalous temperature dependence of the magnetic moments. It has recently been suggested that the increase of the magnetic moment with increasing temperature, which has been reported in several publications, ${ }^{28-30,32,34}$ may be explained by thermoinduced magnetization, which is related to the magnetic moment with the absolute value given by Eq. (18). ${ }^{35}$ It should be emphasized that the states with magnetization up and down are degenerate in zero applied field and the average magnetization is therefore zero. When a magnetic field is applied the degeneracy will be lifted, resulting in a nonzero contribution to the magnetization. Here, we derive expressions for the thermal average of the absolute value of the magnetic moment in zero applied field and the initial susceptibility of nanoparticles of antiferromagnetic materials. For simplicity, we perform the calculations for particles without an uncompensated moment, and we assume that there is no spin canting of surface spins. The derivations show that both quantities increase with temperature in contrast to the magnetization of normal bulk materials.

The absolute value of the thermoinduced magnetic moment is given by Eq. (18). Replacing $\cos \theta_{j}$ by $\left(1-\sin ^{2} \theta_{j}\right)^{1 / 2}(j=A, B)$ in Eq. (18) we find, by use of Eq. (16), for $\delta \ll 1$

$$
\left|\mu_{A F}\right| \simeq M_{S} V \delta \frac{\sin ^{2} \theta_{B}}{\cos \theta_{B}} \simeq M_{S} V \delta \sin ^{2} \theta_{B},
$$

where the last approximation is valid at low temperatures such that only states with small values of $\theta_{A}$ and $\theta_{B}$ are populated. The precession states are characterized by the angles $\quad \cos \theta_{B}=1,1-\xi, 1-2 \xi, \ldots, 1-n \xi, \ldots, 1-(N-1) \xi$, where $\xi$ is defined in Eq. (21). At low temperatures $n \xi \ll 1$ and $\sin ^{2} \theta_{B} \simeq 2 n \xi$. With $\alpha=K V / k_{B} T$, we find, by using a procedure similar to that used in Sec. III, that the thermal average of $\left|\mu_{A F}\right|$ is given by

$$
\begin{aligned}
\left\langle\left|\mu_{A F}\right|\right\rangle & =M_{S} V \delta \frac{\sum_{n=0}^{N-1} 2 n \xi \exp (-2 \alpha n \xi)}{\sum_{n=0}^{N-1} \exp (-2 \alpha n \xi)} \simeq \frac{M_{S} V \delta}{\alpha} \\
& \simeq 2 g \mu_{B} \frac{k_{B} T}{\hbar \omega_{0}} .
\end{aligned}
$$

For $\omega_{0} \simeq 10^{12} \mathrm{~s}^{-1}$ we find that $\left\langle\left|\mu_{A F}\right|\right\rangle \simeq 200 \mu_{B}$ at $300 \mathrm{~K}$. It is remarkable that $\left\langle\left|\mu_{A F}\right|\right\rangle$ is independent of the particle size. With increasing particle size a larger number of atoms will contribute to the magnetic moment. However, concurrently, the higher excited states of the uniform mode will be less populated, because of the larger particle size [see, e.g., Eq. (26)]. The net result is a size-independent magnetic moment.

As there is no energy barrier between states with $\delta<0$ and $\delta>0$, the size and direction of the thermoinduced magnetic moment will fluctuate rapidly such that $\left\langle\mu_{A F}\right\rangle=0$ in zero applied field. We now consider the situation where a magnetic field, $B_{\text {ext }}$, is applied parallel to the easy direction of magnetization. We then have different probabilities, $p^{+}$ and $p^{-}$for the magnetic moment to be parallel or antiparallel to the applied field, respectively, and a nonzero magnetization is induced. The thermal average of the magnetic moment is then given by

$$
\left\langle\mu_{A F}\right\rangle=\sum_{n=0}^{N-1}\left|\mu_{A F}(n)\right| p(n)\left[p^{+}-p^{-}\right],
$$

where $\left|\mu_{A F}(n)\right|$ is given by 


$$
\left|\mu_{A F}(n)\right| \simeq 2 M_{S} V \delta n \xi .
$$

The probability, $p(n)$, that the precession state $n$ is occupied is

$$
p(n)=\frac{\exp (-2 \alpha n \xi)}{\sum_{n=0}^{N-1} \exp (-2 \alpha n \xi)},
$$

and

$$
\begin{aligned}
p^{ \pm} & =\frac{\exp \left( \pm \frac{\left|\mu_{A F}(n)\right| B_{\text {ext }}}{k_{B} T}\right)}{\exp \left(\frac{\left|\mu_{A F}(n)\right| B_{\text {ext }}}{k_{B} T}\right)+\exp \left(-\frac{\left|\mu_{A F}(n)\right| B_{\text {ext }}}{k_{B} T}\right)} \\
& \simeq \frac{1}{2}\left(1 \pm \frac{\left|\mu_{A F}(n)\right| B_{\text {ext }}}{k_{B} T}\right),
\end{aligned}
$$

where the last approximation is valid for small values of $B_{\text {ext }}$. Introducing

$$
F(\alpha)=\ln \left(\sum_{n=0}^{N-1} \exp (-2 \alpha n \xi)\right)
$$

one finds

$$
\left\langle\mu_{A F}\right\rangle \simeq \frac{\left(M_{S} V \delta\right)^{2}}{k_{B} T}\left[\frac{d^{2} F(\alpha)}{d \alpha^{2}}+\left(\frac{d F(\alpha)}{d \alpha}\right)^{2}\right] B_{e x t} .
$$

For small values of $n \xi$ we find that $F(\alpha) \simeq-\ln (2 \alpha \xi)$, and we obtain the initial susceptibility

$$
\chi_{i}=\frac{\mu_{0}\left\langle\mu_{A F}\right\rangle}{V B_{\text {ext }}} \simeq \frac{8 \mu_{0} k_{B} T}{V}\left(\frac{g \mu_{B}}{\hbar \omega_{0}}\right)^{2} .
$$

Equations (28) and (35) show that both the average of the absolute value of the magnetic moment and the initial susceptibility due to the thermoinduced magnetic moment increase linearly with temperature. This is in accordance with experimental studies of the magnetic properties of ferrihydrite $^{28}$ and ferritin nanoparticles. ${ }^{29}$

Using Eq. (25), we find for $T \rightarrow 0$

$$
\frac{\Delta M_{S}}{M_{S}}=\frac{\hbar \omega_{0}}{4 K V} \text {. }
$$

Thus, the sublattice magnetization at $T=0 \mathrm{~K}$ will be reduced in nanoparticles compared to the bulk value. The temperature dependence of $\Delta M_{S} / M_{S}$ is shown in Fig. 2.

It is noteworthy that at $T=0 \mathrm{~K}$ the uniform mode will give a zero-point contribution, $\mu(0)=\frac{1}{2} g \mu_{B}$, to the magnetic moment. The precession angles in the ground state of the uniform mode are given by

$$
\cos \theta_{B} \simeq 1-\frac{g \mu_{B}}{4 M_{S} V \delta}
$$

and

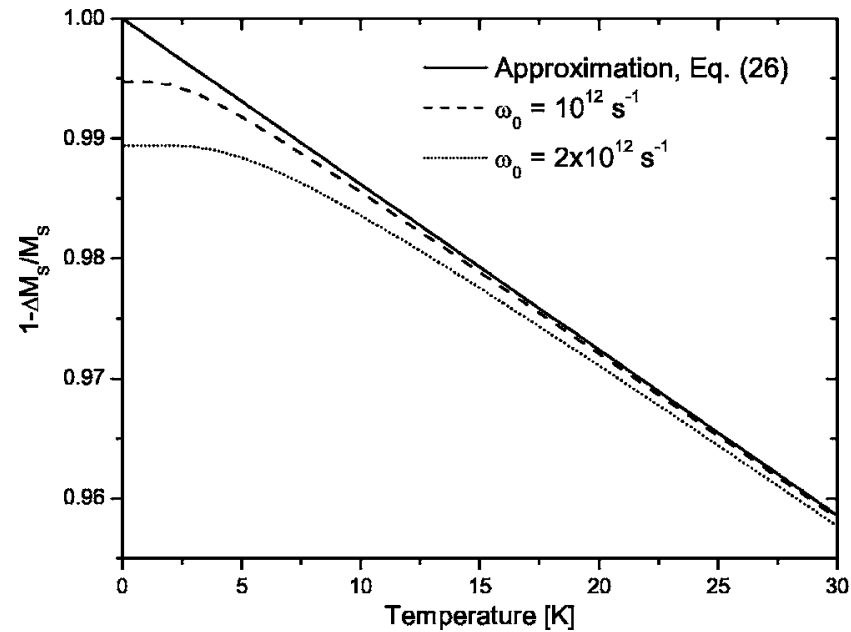

FIG. 2. The temperature dependence of the normalized sublattice magnetization in a nanoparticle of an antiferromagnetic material, calculated by use of Eq. (25) for $K V=5 \times 10^{-21} \mathrm{~J}$.

$$
\cos \theta_{A} \simeq 1-\frac{g \mu_{B}}{4 M_{S} V \delta}(1+2 \delta) .
$$

In bulk materials this contribution to the magnetization can be considered negligible. However, in nanoparticles it may be significant. Thus, antiferromagnetism is, strictly speaking, nonexistent even in nanoparticles with zero uncompensated magnetic moment.

\section{SUMMARY}

Using a spin-wave model to calculate the influence of uniform magnetic excitations on the magnetic properties of nanoparticles, we find that the classical model, which gives a linear temperature dependence of the (sublattice) magnetization, is a fair approximation for nanoparticles of both ferro-, ferri-, and antiferromagnetic materials. For nanoparticles of antiferromagnetic materials, we find that excitation of the uniform mode gives rise to a deviation from the linear temperature dependence at low temperatures. In nanoparticles of antiferromagnetic materials the two sublattices are not strictly antiparallel, and in an applied field this gives rise to a contribution to the magnetization, which increases with temperature. We have calculated the numerical value of this thermoinduced magnetic moment and the related initial susceptibility.

\section{ACKNOWLEDGMENTS}

The work was supported by the Danish Technical Research Council through the framework program Nanomagnetism. The authors are grateful to $\mathrm{C}$. Frandsen for valuable comments. 
*Electronic address: morup@fysik.dtu.dk

†Electronic address: britt@brittrosendahl.dk

${ }^{1}$ J. L. Dormann, D. Fiorani, and E. Tronc, Adv. Chem. Phys. 98, 283 (1997)

${ }^{2}$ J. L. García-Palacios, Adv. Chem. Phys. 112, 1 (2000).

${ }^{3}$ P. E. Jönsson, Adv. Chem. Phys. 128, 191 (2004).

${ }^{4}$ E. L. Nagaev, Phys. Rep. 222, 199 (1992).

${ }^{5}$ X. Batlle and A. Labarta, J. Phys. D 35, R15 (2002).

${ }^{6}$ R. H. Kodama, J. Magn. Magn. Mater. 200, 359 (1999).

${ }^{7}$ L. Néel, Ann. Geophys. (C.N.R.S.) 5, 99 (1949).

${ }^{8}$ W. F. Brown, Jr., Phys. Rev. 130, 167 (1963).

${ }^{9}$ S. Mørup and H. Topsøe, Appl. Phys. 11, 63 (1976).

${ }^{10}$ S. Mørup, J. Magn. Magn. Mater. 37, 39 (1983).

${ }^{11}$ M. F. Hansen, F. Bødker, S. Mørup, K. Lefmann, K. N. Clausen, and P.-A. Lindgård, Phys. Rev. Lett. 79, 4910 (1997).

${ }^{12}$ M. F. Hansen, F. Bødker, S. Mørup, K. Lefmann, K. N. Clausen, and P.-A. Lindgård, J. Magn. Magn. Mater. 221, 10 (2000).

${ }^{13}$ P. V. Hendriksen, S. Linderoth, and P.-A. Lindgård, Phys. Rev. B 48, 7259 (1993).

${ }^{14}$ P. Crespo, J. M. González, A. Hernando, and F. J. Yndurain, Phys. Rev. B 69, 012403 (2004).

${ }^{15}$ H. Kachkachi and D. A. Garanin, Eur. Phys. J. B 22, 291 (2001).

${ }^{16}$ H. Kachkachi and D. A. Garanin, Physica A 300, 487 (2001).

${ }^{17}$ H. E. Stanley, Phys. Rev. Lett. 20, 589 (1968).

${ }^{18}$ H. Thomas, Z. Angew. Phys. 21, 13 (1966).

${ }^{19}$ K. Binder, H. Rauch, and V. Wildpaner, J. Phys. Chem. Solids 31, 391 (1970).
${ }^{20}$ M. J. Klein and R. S. Smith, Phys. Rev. 81, 378 (1951).

${ }^{21}$ S. Mørup, B. S. Clausen, and P. H. Christensen, J. Magn. Magn. Mater. 68, 160 (1987).

${ }^{22}$ A. H. Morrish, The Physical Principles of Magnetism (Wiley \& Sons, New York, 1965).

${ }^{23}$ D. H. Martin, Magnetism in Solids (The MIT Press, Cambridge, MA, 1967).

${ }^{24}$ C. Kittel, Phys. Rev. 82, 565 (1951).

${ }^{25}$ F. Keffer and C. Kittel, Phys. Rev. 85, 329 (1952).

${ }^{26}$ S. Chikazumi, Physics of Ferromagnetism, Second Edition (Clarendon Press, Oxford, 1997).

${ }^{27}$ S. A. Makhlouf, F. T. Parker, F. E. Spada, and A. E. Berkowitz, J. Appl. Phys. 81, 5561 (1997).

${ }^{28}$ M. S. Seehra, V. S. Babu, A. Manivannan, and J. W. Lynn, Phys. Rev. B 61, 3513 (2000).

${ }^{29}$ J. G. E. Harris, J. E. Grimaldi, D. D. Awschalom, A. Chiolero, and D. Loss, Phys. Rev. B 60, 3453 (1999).

${ }^{30}$ S. H. Kilcoyne and R. Cywinski, J. Magn. Magn. Mater. 140144, 1466 (1995).

${ }^{31}$ S. A. Makhlouf, F. T. Parker, and A. E. Berkowitz, Phys. Rev. B 55, R14717 (1997).

${ }^{32}$ D. Vollath, D. V. Szabó, and J. O. Willis, Mater. Lett. 29, 271 (1996).

${ }^{33}$ L. Néel, C. R. Hebd. Seances Acad. Sci. 252, 4075 (1961).

${ }^{34}$ M. Bañobre-López, C. Vázquez-Vázquez, J. Rivas, and M. A. López-Quintela, Nanotechnology 14, 318 (2003).

${ }^{35}$ S. Mørup and C. Frandsen, Phys. Rev. Lett. 92, 217201 (2004). 